\title{
Optimization of plastic foam composition for insulation systems
}

\author{
Anton Pilipenko ${ }^{1,}$, Ekaterina Bobrova $^{2}$, and Alexey Zhukov ${ }^{1}$ \\ ${ }^{1}$ Moscow State University of Civil Engineering, 129337 Yaroslavskoe shosse, 26, Moscow, Russia \\ ${ }^{2}$ NRU High School of Economics, 101000, Myasnitskaya st., 20, Moscow, Russia
}

\begin{abstract}
Construction insulation systems should provide solutions to the problems of creation of suitable conditions of load-bearing construction elements and whole construction, reduction of heat loss through thermal insulation layer, and creation of living comfort in buildings. The article presents the results of experimental studies, the purpose of which is to optimize the composition of extruded polystyrene foam, the formation of methods for selecting its composition and the development of systems for the use of products based on extruded polystyrene foam. Main provisions of the method of analytical optimization, which allows significantly reducing the material and time costs for processing the experimental results, are also provided. Aspects of the implementation of building systems using extruded polystyrene foam both from the point of view of minimizing heat loss through contact areas and the degree of influence of vapor permeability of building structures on the temperature and humidity conditions of premises are studied. Mandatory criteria for construction with the use of building systems are safety conditions, including both structural safety (including maintenance-free cycle) and fire safety.
\end{abstract}

\section{Introduction}

The concept of a building system was introduced into everyday life by both builders and designers in the early 1990s, first for the construction of facades and interiors, and then extended to other building structures. The main feature of the systems and the purpose of their creation is to ensure the joint work of building materials (different in their nature and functionality) in order to increase the efficiency of the structures [1-3]. Insulation systems for building structures imply a solution to the following task groups:

- creation of favorable conditions for the operation of the structure as a whole, which allows maintaining durability for a standard period and extend the maintenance-free periods of operation;

- optimization of heat loss through the insulating envelope implies, firstly, the use of effective thermal insulation materials; secondly, minimization of heat loss through contact heat-conducting bridges; thirdly, the elimination of air and vapor-air mixture filtration through insulating layers. Increase in the thickness of thermal insulation that exceeds the

* Corresponding author: pilipenko.ans@gmail.com 
optimal one does not practically contribute to an increase in the thermal resistance of the insulating sheath. This significantly increases the cost of both materials and installation.

- the creation of comfortable temperature and humidity conditions, which also implies the use of effective thermal insulation [4-6].

\section{Materials and methods}

The usage of gas-filled plastics in plaster facade finishing systems with heat insulation allows increasing the thermal and physical characteristics of the walls (these materials have high moisture resistance and, compared to mineral wool plates for facades, lower thermal conductivity of vapor and air permeability). The use of such products, in particular extruded polystyrene foam plates (XPS-plates), involves the implementation of additional fire prevention measures: constructional (fire-prevention splitting and protection of window and door frame framing) and technological measures - correction of extruded polystyrene foam compositions [7-10].

Analytical optimization of dependencies obtained by processing the experimental results using mathematical statistics allow creating mathematical models that adequately describe technological processes and solve problems of their optimization, including the optimization of the composition of thermal insulation materials, including foamed plastics.

\section{Results}

The conducted studies were made to optimize the consumption of the main components and to develop a method for selecting the composition of XPS plates. The increase in the strength of the products was achieved through the introduction of special fillers-modifiers. The principle of structure modification due to the introduction of fillers or chemical modifiers is widely used in polymer technology. The technological aspect of the implementation of this principle is the optimization of the composition based on the methods of mathematical statistics and active experiment $[10,11]$.

The consumption and specific surface area of the additive modifier, as well as the consumption of the porophore and the activity of the porophore were taken as variable factors. The average density of XPS plates $\left(\mathrm{Y}_{1}, \mathrm{~kg} / \mathrm{m}^{3}\right)$ and compressive strength of plates with $10 \%$ deformation $\left(\mathrm{Y}_{2}, \mathrm{kPa}\right)$ were taken as response functions. The experimental conditions are presented in table 1.

Table 1. Experimental factors.

\begin{tabular}{|c|c|c|c|c|c|}
\hline \multirow[t]{2}{*}{ Factor } & \multirow{2}{*}{$\begin{array}{c}\text { Code, } \\
\mathrm{X}_{\mathrm{i}}\end{array}$} & \multirow{2}{*}{$\begin{array}{l}\text { Medium } \\
\text { value, } \bar{X}_{\mathrm{i}}\end{array}$} & \multirow{2}{*}{$\begin{array}{c}\text { Factor } \\
\text { variable } \\
\text { interval, } \Delta \mathrm{X}_{\mathrm{i}}\end{array}$} & \multicolumn{2}{|c|}{ Factor's value at level } \\
\hline & & & & -1 & +1 \\
\hline Modifier consumption, \% & $\mathrm{X}_{1}$ & 5 & 2 & 3 & 7 \\
\hline $\begin{array}{l}\text { Modifier specific surface, } \\
\mathrm{m}^{2} / \mathrm{m}^{3}\end{array}$ & $\mathrm{X}_{2}$ & 2200 & 600 & 1600 & 2800 \\
\hline Porophore consumption, $\%$ & $\mathrm{X}_{3}$ & 7 & 1 & 6 & 8 \\
\hline Porophore activity, $\%$ & $\mathrm{X}_{4}$ & 1,3 & 0,2 & 1,1 & 1,5 \\
\hline
\end{tabular}

The analytical optimization technique is also developed by NRU MGSU and tested in the study of various building materials technologies and in the implementation of system solutions based on these materials.

The concept of analytical optimization is based on two statements. At first, the obtained mathematical model (in the polynomial form) is adequate to the real process. It describes it 
with an established degree of accuracy. At second, the obtained mathematical model is an algebraic nonlinear function of several variables: with this function, all types of actions can be carried out using the apparatus of mathematical analysis.

In fact, analytical optimization consists in determining the extremals of a function of several variables for each of the variables (for which partial derivatives are obtained for each of the variables and equate them to zero); solving polynomials taking into account the found extremal functions and obtaining regression equations optimized for one or several variables.

Processing the results of the experiment allowed obtaining regression equations that establish the functional relationship between the variable factors and the resulting parameters:

$$
Y_{1}=36+3 X_{1}-7 X_{3}-4 X_{4}+2 X_{1} X_{3}-3 X_{3} X_{4}
$$

at confidence interval $\Delta b=1,8$

$$
Y_{2}=360+60 X_{1}+30 X_{2}-40 X_{3}+24 X_{1} X_{2}+20 X_{2} X_{3}-32 X_{1}^{2} ;
$$

at confidence interval $\Delta b=16$

Analytical optimization. Differentiation of the equation $\mathrm{Y}_{2}\left(\mathrm{X}_{1}, \mathrm{X}_{2}, \mathrm{X}_{3}\right)$ in partial derivatives made it possible to establish an optimization function. Next, the basic polynomials were calculated using the optimization function, optimized equations for $\mathrm{Y} 1$ and $\mathrm{Y}_{2}$ were obtained, and graphical interpretation was carried out, the result of which was made in a form of a nomogram for the selection of optimized compositions (Fig. 1).

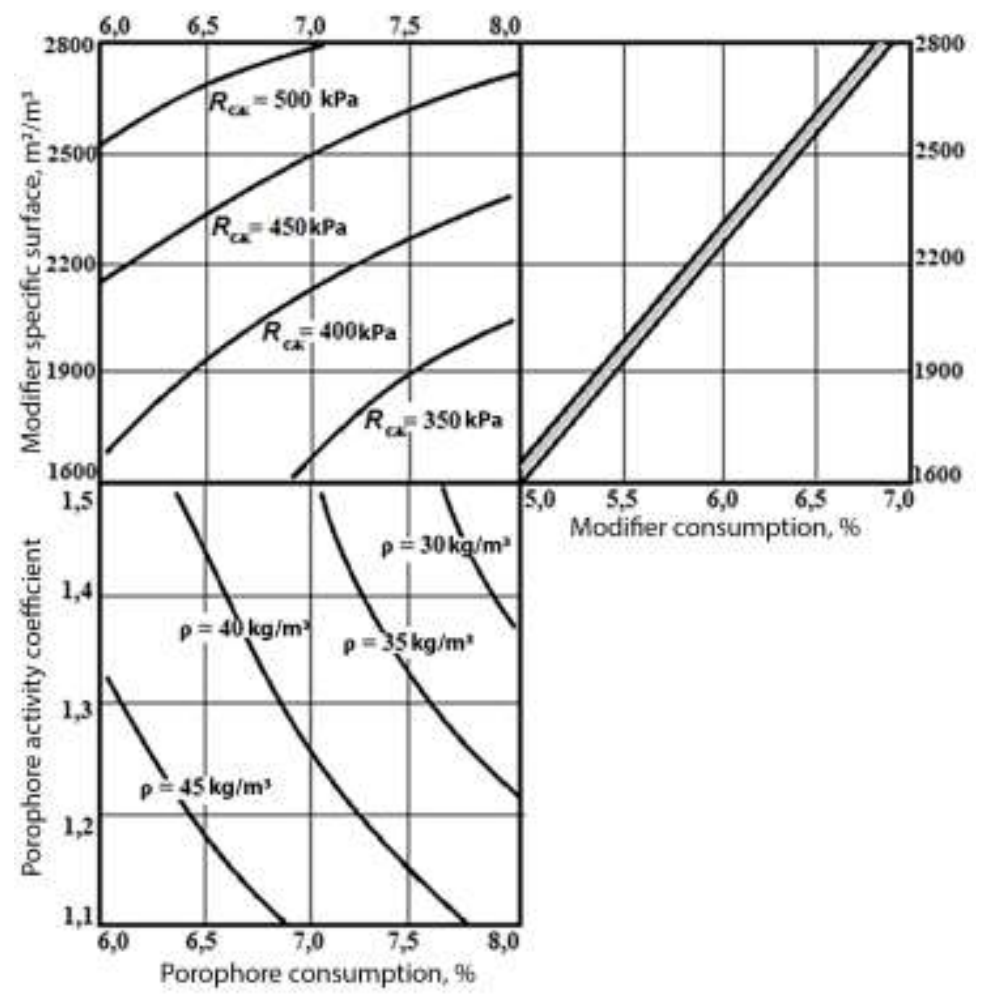

Fig. 1. Nomogram for the selection of the composition of extruded polystyrene foam. 
The engineering interpretation of the dependences obtained allows recommending the following composition for the manufacture of extruded polystyrene foam. Depending on the required strength and the average density of products, the consumption of polystyrene should be $30-40 \mathrm{~kg} / \mathrm{m}^{3}$; porophore consumption $-2-3 \mathrm{~kg} / \mathrm{m}^{3}$; flame retardant $-0.9 \mathrm{~kg} / \mathrm{m}^{3}$.

As a result of the research, correlation dependence was established (with a confidence of $94 \%$ ) between the average density of products and their thermal conductivity (Fig. 2). The results of studies show that in the porosity range of $0.975-0.986$, the function $\lambda(\rho, W)$ has an extremum in absolutely dry products and equilibrium moisture products.

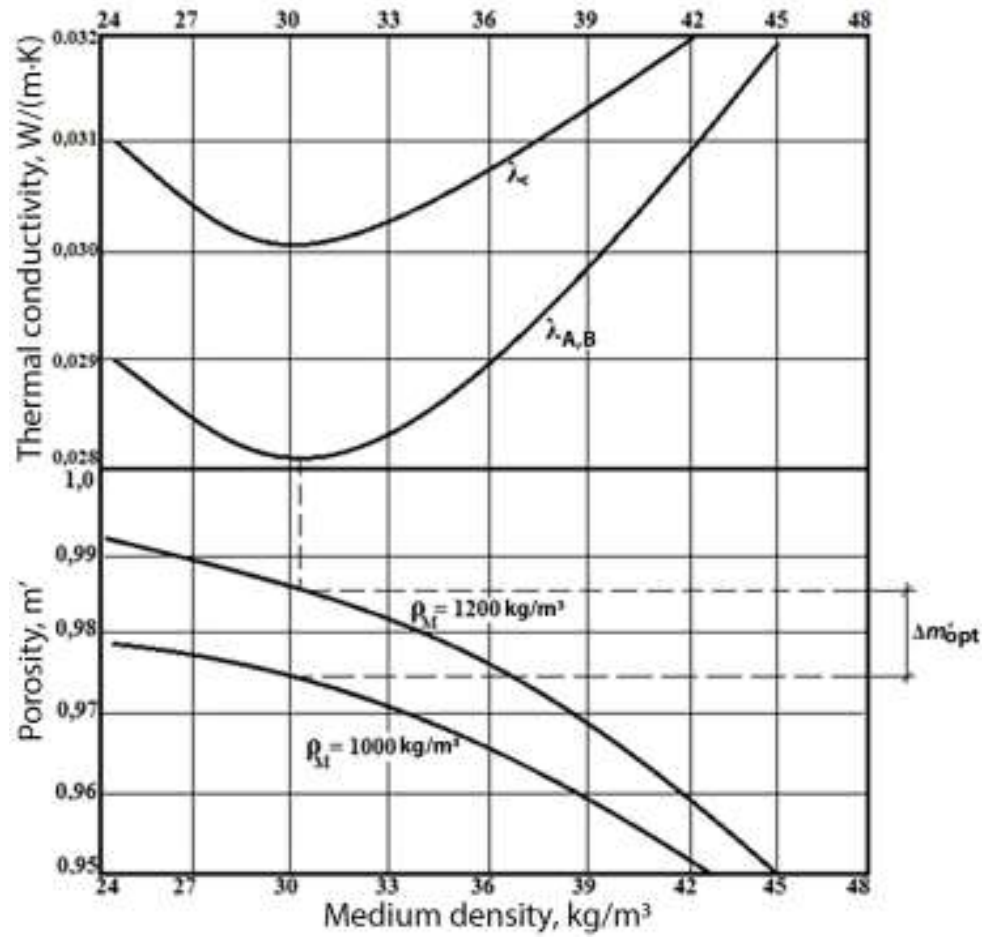

Fig. 2. The dependence of thermal conductivity of extruded polystyrene on the average density and porosity: $\rho_{\mathrm{M}}$ - the average density of the matrix, $\mathrm{kg} / \mathrm{m}^{3} ; \lambda_{\mathrm{c}}, \lambda_{\mathrm{A}, \mathrm{B}}$ - thermal conductivity of a dry material and thermal conductivity for operation zones $\mathrm{A}$ and $\mathrm{B} ; \Delta \mathrm{m}$ ' ${ }_{\mathrm{opt}}$ - optimal porosity interval.

This confirms that irrespective of the type of material, there are such porosity values that correspond to the minimum values of thermal conductivity of products, i.e. the first derivative of the function of the main indicator tends to zero [12, 13]. For highly porous materials such as XPS, the nature of the dependence of thermal conductivity on average density is also important from the point of view of energy efficiency of energy-saving measures. The optimal interval of porosity makes it possible to limit its reduction to a reasonable limit, approaching which leads to a decrease in thermal conductivity, which makes it impractical to manufacture especially lightweight materials.

\section{Discussion}

The use of polystyrene plates significantly reduces the cost of the system of facades of heatinsulation composite. The basis of the system can be bearing, self-supporting and curtain walls of monolithic reinforced concrete, stone and reinforced masonry (density not lower than $600 \mathrm{~kg} / \mathrm{m}^{3}$ ). In the role of fire-prevention cuts and edging, rigid hydrophobic mineral 
wool plates are used on a synthetic binder with stone fibers and a melting point of fibers of at least $1000^{\circ} \mathrm{C}, 40$ to $200 \mathrm{~mm}$ thick, having a compressive strength at $10 \%$ deformation of at least $40 \mathrm{kPa}$ and strength at transverse stretching (tensile strength perpendicular to face surfaces) of at least $14 \mathrm{kPa}$.

XPS plates with a special milled surface are used for thermal insulation of the basement part. This material is characterized by minimal water absorption, high heat transfer resistance and high strength, and the rough surface of the material holds the plaster glue without additional additives.

The base plaster layer plays a protective role in relation to external mechanical and weather effects. Reinforcement of this layer with a mesh increases impact resistance and reduces the risk of cracks. In the composite insulated facade systems, PVC profiles could be used in corners, window trims, expansion joints. They facilitate installation of the system, making the final version more accurate and long-lasting. Decorative plaster also protects the facade from weathering, and as a result, sheds the lifetime of the facade. The structure of the coating is determined by the size and shape of the granular filler used by the tool, as well as by application methods.

In the construction with a thin plaster layer, insulation is attached to the carrier layer of the wall with glue and with additional expansion dowels. When preparing the bearing part of the wall before fixing the insulation to it, it is recommended to use if necessary (depending on the surface condition): antifungal composition, primer. In case thermal insulation is used on the basis of foamed polymers, additional requirements are also imposed on the plaster layers.

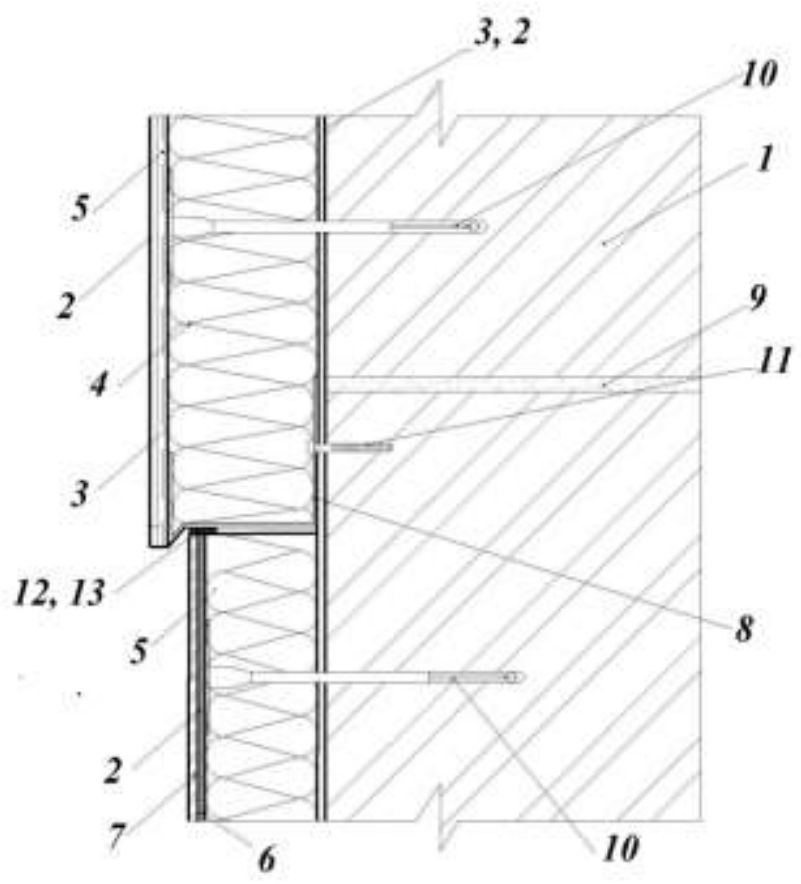

Fig. 3. Facing of the facade in the system using XPS-plates: 1 - base; 2 - impregnating reinforcing soil; 3 - adhesive composition; 4 - heat-insulating layer (cuttings made of stone wool); 5 - heat insulation layer of extruded polystyrene foam; 6 - facade reinforcing alkali-resistant fiberglass mesh; 7 - decorative plaster composition; 8 - starting profile; 9 - waterproofing layer; 10 - anchor with dishshaped polymeric dowel; 11 - dowel and anchor for fastening the profile; 12 - the filled deformation seam; 13 - self-expanding sealing tape. 


\section{Conclusions}

All conditions and requirements for the installation of composite facade insulation systems using thermal insulation on the basis of foamed polymers are regulated by joint ventures SP 293.1325800.2017 (introduced by the Order of the Ministry of Construction and Public Utilities of the Russian Federation of July 10, 2017) and GOST 32494-2013 Interstate standard "Buildings and facilities. The method of mathematical modeling of temperature and humidity regime of enclosing structures".

The implementation of facade insulation systems with the use of foamed plastics allows reducing the construction cost and achieving an additional heat-saving effect due to the lower thermal conductivity of materials and the stability of their properties in humid environments. The minimization of the fire hazard of this type of materials is carried out at the expense of special design and engineering measures. Low vapor permeability of foamed plastics has a slight effect on comfort indicators in interiors, since the main volume of ventilation (vapor-air exchange) falls on natural or forced ventilation of rooms, and not on diffusion of the vapor-air mixture through the layers of the building structure.

\section{References}

1. P.M. Zhuk, A.D. Zhukov, Eco. and ind. of Russia. 4, 52 - 57 (2018)

2. I. Gimenez, M.-K. Faroog, A. El Mahi, A. Kondrotas, M. Assarar, Mat. Sci. (Mediagotyra) 10, 34-39 (2004)

3. S.E. Shmelev, Build. Mat. 3, 7-9 (2013)

4. N.P. Umnyakova, V.M. Tsygankov, V.A. Kuzmin, Hous. Constr. 1-2, 38-42 (2018)

5. I.V. Bessonov, A.V. Starostin, V.M. Oskina, Vestnik MGSU. 3, 134-139 (2011)

6. V.G. Gagarin, V.V. Kozlov, Acad. 2, 60-63 (2006)

7. I.J. Gnip, V.J. Keršulis, S.J. Vaitkus, Mech. of Comp. mat. 41(4), 357-364 (2005)

8. I.J. Gnip, V.I. Keršulis, S.J Vaitkus, Constr. Mat. 12, 40-44 (2012)

9. A.D. Zhukov, K.A. Ter-Zakaryan, A.V. Zayafarov, Ye.S. Petrovsky, D.U. Tuchaev, Roofs and roof mat. 6, 27-29 (2017)

10. V.S. Semenov, T.A. Rozovskaya, A.Yu. Gubsky, Build. Mat. 6, 21-24 (2016)

11. Y. Wang, Z. Huang, L. Heng, Int. J. of Proj. Mngt 25(2), 143-149 (2007)

12. A.D Zhukov, V.S Semenov, I.J. Gnip, S.J. Vaitkus, Proceedings of the XXVI R-S-P Seminar, Theoretical Foundation of Civil Engineering 117(24) (2017)

13. A.D. Zhukov, N.V. Naumova, R.M. Mustafayev, N.A. Mayorova, Ind. and Civ. Constr. 7, 48-51 (2014) 